\title{
Analyzing NIH KL2 outcomes: A pilot study using administrative data
}

Matthew VanEseltine ${ }^{1 *}$, Nancy Calvin-Naylor ${ }^{1}$, Jason Owen-Smith ${ }^{2}$

${ }^{1}$ Institute for Research on Innovation and Science, Institute for Social Research, University of Michigan, Ann Arbor, Michigan, United States of America

${ }^{2}$ Department of Sociology and Institute for Research on Innovation and Science, University of Michigan, Ann Arbor, Michigan, United States of America

* Corresponding author

Email: matvan@umich.edu (MVE) 


\section{Abstract}

\section{Background}

The U.S. National Institutes of Health (NIH) funds "K" awards that provide both resources and access to mentoring believed to be invaluable for early career faculty. The KL2 Mentored Career Development Award trains early-career clinicians with the goal of guiding scholars toward an independent clinical and translational research career. This study presents the pilot of a systematic, low-burden method to examine scientific and career outcomes for these awardees, applying a novel set of linked administrative data.

\section{Methods}

Clinical and Translational Science Award hubs administering KL2 awards at ten universities who participate in the Institute for Research on Innovation and Science (IRIS) provided names of scholars in their KL2 cohorts. Using extensive data on sponsored projects which IRIS member universities provide, we linked the KL2 scholars to information on subsequent publication, patent, and grant activity.

\section{Results}

Analyses of linked data supported a rigorous, sustainable, low-cost approach to examining career outcomes. A subset of key metrics identified by CTSA evaluators were operationalized as an examination of the post-award careers of KL2 awardees. We successfully identified contemporaneous faculty with different NIH K Awards to use as comparison groups. The pilot culminated in university-specific and aggregate reporting to all participating hubs.

\section{Conclusions}

This pilot demonstrates that substantive evaluations of early career programs are possible using administrative data from universities with low additional burden. Integration of research on 
career development outcomes offer new means to examine the effects of increasingly diverse funding, team, and collaborative network structures, advancing both knowledge about the workings of science and practices to support early career faculty. This approach could be extended to support rigorous multi-institutional evaluation and research on a range of student and faculty training mechanisms.

\section{Introduction}

The commitment of resources to support the next generation of faculty researchers necessitates a systematic examination of the effects on their scientific outcomes and career trajectories. Administrative data curated by the Institute for Research on Innovation \& Science (IRIS) enables analyses of scientific outcomes, team structures, and career trajectories for specific groups of researchers. We present a pilot project from IRIS that pertains to nascent clinical faculty with a research career development award from the NIH with the goal of demonstrating an innovative approach and discussing the utility of such data in evaluating the effectiveness of such programs as well as strategic decision-making.

\section{KL2 Mentored Career Development Awards in the Clinical and}

\section{Translational Science Award program}

The National Institutes of Health (NIH) established its Clinical and Translational Science Award (CTSA) in the early 2000s in an effort to accelerate the application of basic science research findings to human health. Administered by the National Center for the Advancement of Translational Science (NCATS), the CTSA program is a national consortium of institutions (or hubs) that works to "catalyze innovation in training, research tools, and processes" [1]. Federal 
investment in clinical and translational activities is substantial; for example, in 2019, Congress allocated almost \$560 million to NCATS for its CTSA flagship program [2].

A key goal of the CTSA program is to train a translational workforce, and therefore all medical research universities and institutions with a CTSA award have a KL2 Mentored Career Development Award program targeting clinicians who have recently completed professional training. KL2 appointees are drawn from a variety of health-related fields, such as medicine, dentistry, and epidemiology. The KL2 award provides mentorship, training, and release time to pursue a research agenda. The KL2 falls under the auspices of the NIH's Research Career Development (K) programs, a broad set of awards that are intended to provide research and training opportunities for undergraduate, graduate, and postdoctoral scholars [2].

Measuring the impact of CTSA training programs presents substantial challenges. The potential range of professional and scientific outcomes of post-K scholars is broad. Surveys of busy researchers require considerable time and effort spent by administrators to overcome low response rates and researcher mobility. The resulting data suffer from the well-documented methodological limitations of self-reported questionnaires [3-5]. When follow-up survey information is successfully gathered, the lack of comparable data from appropriate reference groups poses significant challenges to rigorous evaluation.

An internal CTSA study and an external evaluation by the Institute of Medicine (IOM) concurrently conducted around 2013 raised concerns with the dominant mode of CTSA program assessment generally, including KL2 training efforts [6,7]. Without standardized metrics by which to measure outcomes, institutions had developed their own idiosyncratic, site-specific practices and self-evaluations. A key recommendation of the report was the formalization of these evaluation processes $[1,7]$. Subsequently, NCATS launched the Common Metrics Initiative 
to improve the consortium's capacity for benchmarking, systematic evaluation, and aggregated analyses across the whole range of CTSA activities [8-10]. CTSA working groups have labored to produce clear and consistent sets of metrics. The "Careers in Clinical and Translational Research" metric was among the first to be implemented, much of which directly focuses on the KL2 award [11-13].

\section{Previous evaluations of KL2 training outcomes}

The primary purpose of the KL2 award is to provide tangible career benefits to cohorts of early-career clinical and translational researchers at institutions with CTSA awards (also termed CTSA hubs). Existing quantitative assessments of KL2 scholars have focused on traditional markers of scientific career outcomes, especially publications and grants. Although the use and interpretation of citation counts and rates have been debated for several decades [14-16], they are well-understood measures that may be reasonably practical in the CTSA space [17]. Records of grants achieved following the $\mathrm{K}$ award are similarly useful, as the " $\mathrm{K}$ to $\mathrm{R}$ " transition to independent research funding (exemplified in receipt of an R01 Research Project Grant) is often considered a key indicator of success in KL2 training programs [18-20].

The primary large-scale effort to systematically track KL2 outcomes is the Rockefeller University Graduate Tracking Survey System, which supplements scholar survey data with secondary datasets on grants and publications $[21,22]$. While more consistent and comprehensive data collection is an improvement over idiosyncratic single-institution follow-ups, its creators underscore the lack of a comparison group against which to benchmark. On a smaller scale, an interuniversity assessment of KL2 scholars observed success in attainment of independent research grants, but similarly lacked groups against which that success could be measured [23]. A study within a single CTSA hub to contrast scientific outcomes of its KL2 scholars with other 
$\mathrm{K}$ awardees at its institution overcame the comparison issue [24], but most hubs do not have these data readily available. An alternative approach is needed to fully address the need for systematic, consistent outcome tracking and rigorous comparison groups in evaluating the KL2 scholar program.

\section{Administrative data at the Institute for Research on Innovation and}

\section{Science}

Universities amass large amounts of data about grants they receive, expenditures they make, and people they educate, employ, and serve. They increasingly turn to these data to tell their stories of impact [25-27]. Although administrative datasets are not designed for evaluation or research, they include quantities of information and details of organizations not available elsewhere. The Institute for Research on Innovation and Science (IRIS) is a national initiative building data that can tackle data challenges such as those faced by the CTSA. IRIS is a consortium of universities working to create the data-driven foundation for national science policy research [28]. Each university submits transaction-level administrative records on grantfunded projects to IRIS, which integrates these data into an IRB-approved data repository. This repository links to extensive external data sources to support research and reporting that can explain and improve the public value of universities. IRIS and its partners link these rich research expenditures data to scientific and economic outcomes. One key partner, the U.S. Census Bureau, merges data collected by IRIS to federally restricted data on employment and earnings. Data resulting from this work are made available for research use through multiple outlets, including the Federal Statistical Research Data Center System [29] and a virtual data enclave maintained at the University of Michigan. The potential of these integrated data to gain 
insight into and improve biomedical workforce development led a National Academies of Sciences panel to "strongly urge that research institutions join the IRIS initiative." [30]

The core IRIS dataset, UMETRICS (Universities Measuring the EffecTs of Research on Innovation, Competitiveness, and Science), offers new possibilities for analysis of large scale, multi-institutional research and training investments [27]. IRIS is uniquely positioned to assess programs like the CTSA by using existing administrative data to identify scientific and professional outcomes of translational researchers and programs. Since its establishment in 2015, IRIS membership continues to expand, now including data from 38 universities. IRIS aims to include roughly 150 research universities that would represent some $90 \%$ federal spending on U.S. academic research. This target expansion would include all current CTSA hubs. At present, IRIS member universities report $\$ 99$ billion in research spending, representing about $41 \%$ of all U.S. academic research according to the 2018 Higher Education Research and Development (HERD) survey. IRIS expands these data through record linkage to address key aspects of the process, products, social and economic impact of academic research.

The unique IRIS infrastructure is being leveraged to produce extensive research and reporting. Information on grant employment undergirds analysis of collaboration networks and research teams, while titles and abstracts of grants linked from the Federal RePORTER have already been employed in natural language processing analysis of research topics $[31,32]$. Data on publications, patents and dissertations provide insight into scientific productivity and impact [33,34]. With linkages to the U.S. Census Bureau and other public and restricted sources, estimates of economic impact can be produced offering novel possibilities for workforce and career analyses [35-40]. This network of data provides IRIS with unique insight into scientific careers, particularly in grant-heavy fields such as the biomedical research of the CTSA. 


\section{The present pilot study}

This manuscript reports on a pilot study of career and scientific outcomes of KL2 scholars at 10 CTSA hubs located at IRIS member universities. The interconnected data at IRIS have the potential to contribute to comprehensive, low-cost analyses on federal research grants and scientific outcomes at the individual level. Here, we assess the feasibility of this process for CTSA hubs and their KL2 Mentored Career Development Award programs. Because this approach relies on existing administrative data, this pilot placed little additional burden on CTSA hubs. Through the pilot, we evaluate this process in three necessary objectives:

1. Record linkage between information provided by CTSA hubs about KL2 scholars and university employees captured in the UMETRICS data.

2. Construction of suitable comparison groups of non-KL2 faculty employees.

3. Analysis of scientific outcomes creating useful metrics within and between groups. Each objective is required to create a baseline analytic framework upon which more detailed and comprehensive systems of evaluation could be built using the IRIS infrastructure. If any one of these steps proved inadequate, the pilot would not support further investment in this application of the system.

\section{Materials and methods}

\section{Data sources}

This manuscript reports on a pilot study working with IRIS data and linkages to create an analysis of KL2 scientific and career outcomes among 10 CTSA hubs that are located at IRIS member universities, listed in Table 1. To minimize the burden of participation, we asked for 
only a small amount of data: each CTSA hub provided the names and starting years of each awardee in their KL2 programs. From these hubs we received a starting KL2 sample of 302 scholars.

Table 1. CTSA hubs participating in the pilot.

\begin{tabular}{ll} 
University & Hub \\
\hline Boston University & Clinical \& Translational Science Institute \\
Northwestern University & Northwestern University Clinical and Translational Sciences Institute \\
The Ohio State University & Center for Clinical and Translational Science \\
The Pennsylvania State University & Clinical and Translational Science Institute \\
University of Iowa & Institute for Clinical and Translational Science \\
University of Kansas & Frontiers: University of Kansas Clinical and Translational Science Institute \\
University of Michigan & Michigan Institute for Clinical \& Health Research \\
University of Pittsburgh & Clinical and Translational Science Institute \\
University of Wisconsin-Madison & Institute for Clinical and Translational Research \\
Washington University in St. Louis & Institute of Clinical and Translational Sciences \\
\hline
\end{tabular}

\section{UMETRICS award and employment records}

Moving from a list of names to the planned analyses required extended, linkable information as well as a pool of scholars without KL2 awards from which to build comparisons. The UMETRICS dataset is built from records IRIS receives from its member universities including transaction-level information on all direct cost expenditures for wages, vendor purchases, and subcontracts from federal sponsored research. Payments from each research grant to faculty, staff, postdoctoral trainees, and student employees are documented, and details on subaward payments connect research across institutions. These spending data are standardized across institutions at the level of university $\times$ award $\times$ employee $\times$ pay period. Identifying information at the organization (vendor, sub-awardee) and individual (employee) levels 
facilitates matching to other datasets that can provide information about professional and scientific outcomes. The IRIS UMETRICS 2020 Data Release includes information on 442,945 sponsored awards and 720,679 employees across 72 campuses [41]. In the 10 universities included in this pilot, UMETRICS details 175,029 awards and 295,894 employees.

Analyses that connect $\mathrm{K}$ scholars to research-driven outcomes require data beyond the university records included in UMETRICS. We supplemented UMETRICS with data from several federal sources, each providing different insights into facets of research careers: funding for research, scholarly publications, and patenting.

\section{NIH RePORTER award information}

Although UMETRICS tracks payments from sponsored awards to employees, it does not include details about the roles that those employees have. The role of principal investigator (PI) is of special importance for this pilot. Because the KL2 is an NIH award, many $\mathrm{K}$ scholars continue an NIH funding trajectory, and NIH publicly documents research projects, we focus on $\mathrm{NIH}$ awards in this pilot. Extensive information on NIH-funded research is available through the federal NIH RePORTER (Research Portfolio Online Reporting Tools) system [32]. We use these data to identify the types and timings of awards led by K scholars.

\section{PubMed bibliographic data}

Publications are a well-documented, standard result of scientific research. Given the biomedical focus of KL2 scholars we rely in this pilot on the U.S. National Library of Medicine's PubMed [42] database to find and characterize the publication record of K scholars. MEDLINE, the primary data source in PubMed, indexes over 25 million articles from over 5,000 journals, predominantly scholarly publications in biological and biomedicine fields. 


\section{USPTO patent records}

Although publications are the primary outlet for disseminating scientific information, the implementation of new technologies is often represented in patent activity. These patents are another key scientific outcome for this pilot, as the registration of a patent could indicate a "successful translation" from basic science to practice [43]. PatentsView was established in 2012 as a comprehensive research platform on patents registered with the U.S. Patent and Trademark Office (USPTO) [44]. Building from the original source of USPTO records, PatentsView constructs and provides access to a disambiguated relational database in which patents associated with specific individuals and specific institutions can be located.

\section{Methods}

\section{Record linkage}

To connect these disparate data sources into cohesive analyses, several stages of record linkage are required. Here we give an overview of the connections and fields used to make these links; the success of these linkages is described in the Results section below.

The first critical data linkage was the match of CTSA scholars to UMETRICS employees. Although the sample of KL2 scholars provided by hubs was relatively small, the population of employees being paid on federal grants at each university is much larger. Due to the sheer number of potential matches, we employed a combination of automated and manual methods. We first assessed all possible scholar $\times$ employee pairs within each university using Jaro-Winkler string comparisons, then reviewed all highest-scoring proposed matches by hand to ensure the best available linkage for each.

The second set of critical data linkages connect UMETRICS to additional data, allowing us to link CTSA scholars transitively to these external sources. IRIS has created and maintains 
regular updates to these independent of this pilot. Once a $\mathrm{K}$ scholar has been identified as a UMETRICS employee, their employee transaction record provides a list of every federally sponsored award payment to that scholar at that institution in the covered time period. With a combination of the scholar's name and affiliated awards, IRIS maintains linkages to each additional dataset described above. UMETRICS federal awards are linked to NIH RePORTER awards by comparing university-provided award numbers to the Federal Award Identification Number (FAIN) of the award in RePORTER records. PubMed publication records include linked FAINs cited in the research, and so building on this UMETRICS-NIH award crosswalk, we can identify publications that share awards with K scholars. We then compare K scholar names to these linked publication records to identify authorship and position. Finally, IRIS maintains a linkage between UMETRICS employees and inventors in the PatentsView database using a fullname string comparison conditional on a match between the university of employment and the patent assignee organization.

\section{Operationalization of outcomes}

Building on the work of Rubio and colleagues [6] and the CTSA Common Metrics Initiative [10], we identified six key outcomes which we operationalize in eight distinct measures. The alignment of these outcomes to CTSA metrics and their data sources in IRIS is

outlined in Table 2. This study is by no means a comprehensive evaluation of these metrics but, rather, uses the individual outcomes of KL2 researchers to address key components of CTSA goals. 
Table 2. Alignment and operationalization of CTSA metrics.

\begin{tabular}{|c|c|c|c|c|}
\hline Concepts & CTSA Metrics & Outcomes & Measures & Data Sources \\
\hline \multirow{3}{*}{$\begin{array}{l}\text { Scientific } \\
\text { productivity }\end{array}$} & \multirow{3}{*}{$\begin{array}{l}\text { Careers } \\
\text { Products }^{\mathbf{1}}\end{array}$} & Ongoing research career & Academic job title & UMETRICS \\
\hline & & Publications & $\begin{array}{l}\text { Publication counts } \\
\text { Frequent journals }\end{array}$ & $\begin{array}{l}\text { NIH RePORTER } \\
\text { PubMed MEDLINE }\end{array}$ \\
\hline & & Patents & Counts & USPTO \\
\hline \multirow[t]{3}{*}{$\begin{array}{l}\text { Transition to } \\
\text { independence }\end{array}$} & \multirow{3}{*}{$\begin{array}{l}\text { Careers } \mathbf{1}^{\mathbf{1}, 2} \\
\text { Economic return } \\
\text { Collaboration }^{2} \\
\text { Products }^{2}\end{array}$} & Achievement of R01 & Payment as PI on R01 & $\begin{array}{l}\text { NIH RePORTER } \\
\text { UMETRICS }\end{array}$ \\
\hline & & Age at/time to at R01 & $\begin{array}{l}\text { Age at first R01 } \\
\text { Age at K and first R01 }\end{array}$ & UMETRICS \\
\hline & & Scientific teams & $\begin{array}{l}\text { Occupational classes } \\
\text { paid on first } \mathrm{R} 01\end{array}$ & UMETRICS \\
\hline
\end{tabular}

${ }^{1}$ Common Metrics Initiative [8]

${ }^{2}$ Evaluation Key Function Committee [6,9]

A fundamental goal of the KL2 program is to enable scholars to cultivate a leading biomedical research agenda over the course of their careers [45]. The guidelines for the "Careers in Clinical \& Translational Research" Common Metric identify several ways in which KL2 scholars can be determined to be engaged in research. Two data points in UMETRICS can be used to make this determination: job titles can be examined to identify those indicative of a research career, and subsequent grant attainment can be tracked. We also include traditional markers of scientific productivity such as publications [17] and patent activity.

Prior studies of CTSA KL2 programs $[6,8,11,12]$ point to the importance of moving from a mentored research program to an independent research career, typically represented by attainment of an independent investigator award from organizations such as the NIH and PCORI. For the purposes of this pilot, we focus on the achievement of an NIH Research Project (R01) as an indicator of an independent research career. IRIS tracks this outcome of transition to independence by examining whether and when $\mathrm{K}$ awardees attained an R01 award after the 
mentored training program, the average age of award attainment, and time between the start of the mentored award to the start of the independent award. Finally, collaborating with colleagues is a hallmark outcome for CTSA training programs and translational science generally [7]. IRIS can identify markers of team science endeavors, including team size and composition of an R01 award after the mentored training program.

\section{Results}

We assess the results of this pilot project based on the success of three objectives outlined above: (1) linkage between KL2 scholars in UMETRICS data; (2) construction of appropriate comparison groups; and (3) analyses of the outcome metrics of interest.

\section{Linking CTSA KL2 scholars to UMETRICS employees}

The first step toward an analysis of outcomes was to find CTSA scholars as employees in the UMETRICS data. Without this linkage, the planned analyses would be impossible. Linkage between the cohort information provided by hubs and employee transactions was designed to be straightforward. Every UMETRICS employee payment is accompanied by a sponsored award number, and so the list of potential name matches at a university (which might otherwise produce many false positives with common names) can be narrowed greatly by restricting the match to a

specific award. In this case, we expected that every KL2 scholar would be paid on a KL2 award, narrowing the thousands of employees at each university to a small handful.

We conducted preliminary record linkage using 302 names submitted from nine hubs (the tenth would join the pilot after this preliminary linkage). Restricting the employee sample to KL2 payments, a Jaro-Winkler similarity score of 0.9 to match scholar to employee was 
supported by comprehensive clerical review of the results. In this first run, 231 KL2-paid employees were linked to KL2 scholars, a 76.5\% match rate, which was somewhat lower than we anticipated. We examined the UMETRICS award and employee data and discussed these findings with the nine CTSA hubs, arriving at several possible conclusions why a small percentage of KL2 scholars were not matched:

1. Partner institutions: Some CTSA programs offer KL2 awards to scholars at partnering institutions, subcontracting to those partners for salaries. These scholars do not appear as employees in the university that hosts the CTSA hub. Without expansion of IRIS membership to partner institutions, these scholars cannot be included in a UMETRICS-based analysis.

2. Pending UMETRICS updates: CTSA hubs provided us their latest data, but at the time of linkage not every hub's university had updated UMETRICS data to include that cohort, right censoring all such scholars. This issue is remedied over time, as most universities submit annual updates.

3. Non-sponsored/institutional funding: The KL2 is always a salaried award, but in discussion with hubs we learned that, for varying reasons, scholars might occasionally be paid from internal accounts, although this is an unusual circumstance. Because UMETRICS data are built from records of federally sponsored research, scholars who were paid only on non-sponsored or internal university accounts could not be captured in this pilot.

4. Name changes: Legal changes in names, name variants/nicknames, and varying classification of names (e.g., middle names as first names or combined last names) can lead to substantially different recorded names; with too large a change, the name 
may fall outside the reasonable range of string distance used in the preliminary match criteria.

Of these concerns, only the last could be quickly remedied with additional information from hubs. In addition, it also was not possible to determine which of these potential circumstances were most (or least) plausible in accounting for non-matched KL2 scholars. Expanding to highsimilarity name matches with employees never paid on KL2 awards yielded an additional 26 matches, for a total of 257 and an $85.1 \%$ match rate.

Before the final match, additional name details and cohort information were requested from the nine initial hubs, and a tenth hub joined the pilot bringing 15 new names, of which 13 were matchable. Updates from the nine existing hubs added 21 names (many from fresh cohorts), 11 of which could be matched. The ultimate list of 338 hub-provided names were successfully matched to 281 employees, for a total match rate of $83.1 \%$. Of the successful matches, five scholar awards were too recent to be covered by the university's UMETRICS data, yielding a negative possible follow-up time, and so were excluded from subsequent analyses. The final sample was thereby $276 \mathrm{KL} 2$ scholars, meaning that $81.7 \%$ of the provided names could be successfully linked into the UMETRICS data. Ideally, we could locate every KL2 scholar in the UMETRICS data, but this match rate was sufficiently high that we had confidence we could reflect the outcomes of a comfortable majority of KL2 participants.

\section{Constructing comparison groups of $\mathrm{K}$ scholars}

Without a suitable comparison group, most statistics we gather would not go beyond the kinds of information that CTSA hubs often collect themselves (albeit with additional costs of time and resources) through surveys of KL2 scholars. In consultation with participating hubs, we selected a subset of NIH K awards as eligible for comparison: the K01, K08, K12, and K23. 
Names of these awards, along with the final distributions of the comparison groups, are provided in Table 3. In addition to being the most populous of the awards, these were familiar groups to the hubs, and many of these K awardees, like the KL2s, are focused on clinical or patientoriented work. The aim of the comparison group is not to be identical to the KL2 group, but rather to offer a recognizable quantification of the comparisons being made by CTSA hubs.

Table 3. Distribution of KL2 and K-comparison Scholars.
\begin{tabular}{llr} 
Group & NIH Grant Program & n \\
\hline KL2 Scholar & KL2 Mentored Career Development Award & 276 \\
\hline Comparison & K01 Mentored Research Scientist Career Development Award & 300 \\
Scholar & K08 Mentored Clinical Scientist Research Career Development Award & 362 \\
& K12 Clinical Scientist Institutional Career Development Program & 120 \\
& K23 Mentored Patient-Oriented Research Career Development Award & 257 \\
\hline Total & & 1,315
\end{tabular}

These 1,039 comparison scholars were found by filtering the UMETRICS data to capture faculty and other clinical researchers who were paid in UMETRICS data on a potential comparison K. Each of the K awards in this pool was linked to NIH award data. Because the K12 is an institutional award, K12 employees who were K PIs were excluded from the comparison pool. For the individual awards of the K01, K08, and K23, on the other hand, PI status of the scholar was required. To assess the general plausibility of the comparison group, we examined several characteristics of the groups. The typical ages of KL2 and comparison scholars were comparable (averaging 36.8 and 38.0 years, respectively). $87.7 \%$ of KL2 scholars and $91.8 \%$ of comparison scholars held a faculty job title in their award year, the majority of which in both groups were variants of "Assistant Professor." Average windows of observation from the start 
date of the K award through the final university employment observation in UMETRICS were 85.5 months for KL2 awardees and 101.4 months in the comparison group.

Altogether, this phase of the pilot successfully built a comparison group of K awardees from multiple non-KL2 awards. Using the selection criteria discussed with participating hubs, we found more than three K comparison scholars for each KL2. The groups do have observable differences in the aggregate, such as the longer follow-up among the comparison group. In a more in-depth analysis, these would be incorporated into a modeling strategy and assessed further. Fulfilling the aims of this pilot, the comparison group is recognizably similar in NIH affiliation and stage of research career, thereby meeting the interests of the participating CTSA hubs.

\section{Analyzing scientific outcomes to create useful metrics}

Having successfully linked KL2 scholars, and with an identified group of comparison K scholars at each institution, the final step is to illuminate the early research careers of both groups. Working with participating hubs and established CTSA metrics, we identified a useful set of outcomes focused on early indicators of research career progress (see Table 2 above). We next give an overview of each record linkage that produces the information required to measure each metric before presenting the main product of the pilot, the KL2 Impact Report.

\section{Linkage to NIH, MEDLINE, and USPTO data}

By examining outcomes like publication, patent, and grant activity, we can begin to measure how KL2 and other K scholars develop their early careers. The data at the heart of UMETRICS — university records on federal research awards — provide only a narrow window into career development. Linking employment to external data is a critical step of this pilot. Building the comparison group already employed the UMETRICS/NIH award linkage, as 
eligibility was contingent on being a PI (for the K01, K08, and K23) or not being a PI (for the K12). Because only PI names are included in NIH RePORTER data, and not those of other project team members, our key linkage to NIH RePORTER operates in two stages: first, connect UMETRICS award records to NIH award records; second, identify which (if any) employees on that award are PIs. $44.2 \%$ of KL2 awardees and $62.4 \%$ of K comparison scholars were successfully linked to at least one NIH award (not including the identifying K award).

The full set of paid NIH awards in UMETRICS also provide an opportunity for an awardlinked publication measure. PubMed's MEDLINE publication records include a crosswalk to $\mathrm{NIH}$ awards cited as contributing to publications. By capturing the set of NIH awards that our K sample is PI and/or paid on, we can link to MEDLINE records to create a database of NIH-linked publications for each $\mathrm{K}$ awardee. The scholar's name can then be compared to each author list to determine presence and order of authorship. Although this necessarily leaves out publications with no connection to $\mathrm{NIH}$, this sample does share at least one established connection to $\mathrm{NIH}$ funding in the K award itself. $81.2 \%$ of KL2s and $91.1 \%$ of comparison scholars were linked to at least one post-K publication via this method, with an overall average of 14.5 linked publications per scholar. Although we do not have a ground truth comparison for the number of expected linked publications, these numbers suggested some level of success.

Finally, USPTO data provided patenting information when available for each $\mathrm{K}$ awardee in our sample. For this, we rely on an existing supplementary patent linkage developed by IRIS, which has been shown to successfully link the inventors of $62 \%$ of university patents [46]. For each of these match procedures, the ideal result can be hard to quantify; however, to the extent that these linkages might be inadequate, there is no reason to expect that KL2 or comparison K awardees are differentially affected. That is, if KL2 patents are missed, we expect K awardee 
patents to be missed at approximately the same rate. The same is true for new NIH funding and publications. Attained results did not violate expectations of participating hubs on review; this, too, supports the face validity and success of these linkages.

\section{KL2 Impact Reports}

The culmination of this pilot was the construction and release of a customized KL2 Impact Report to each participating hub. S1 Appendix contains a static version of the complete aggregate report. The dynamic version, available to hubs through an online IRIS Portal, includes interactivity such as customizable comparison subgroups, adjustable date ranges, and additional detail accessible through mouse hover. Each hub was given access to both the aggregate report discussed here and their own university-specific version covering only those $\mathrm{K}$ scholars at their own institution. The KL2 Impact Report consists of five major sections: (1) careers in research,

(2) scientific productivity, (3) R01 attainment, (4) funding sequences, and (5) team science.

Below we briefly highlight three sets of results: publication bibliometrics, funding pathways, and R01 team comparisons.

Table 4. NIH-linked publication comparisons of KL2 and comparison scholars.

\begin{tabular}{lcc} 
Outcome & KL2 & Comparison \\
\hline Average publication count & 9.6 & 15.7 \\
Average co-authors per publication & 8.9 & 8.3 \\
Author position: first & $28.1 \%$ & $26.1 \%$ \\
Author position: last & $26.4 \%$ & $31.5 \%$ \\
\hline $\mathrm{N}$ & 276 & 1,039
\end{tabular}

The major outcomes of the NIH-linked publication report are presented in Table 4. Considerably fewer publications were found for KL2 scholars (9.6) compared to the other K awardees (15.7). We observed a comparable number of average co-authors per publication (8.9 
and 8.3). Although the publication count advantage is clearly with the comparison group, KL2 and comparison scholars appear similar in attainment of key authorship markers, with $28.1 \%$ of $\mathrm{K}$ awardees and $26.1 \%$ of the comparison Ks publishing a first-authored publication post-K and $26.4 \%$ to $31.5 \%$, respectively, publishing with a last authorship (excluding sole-authored articles).

Fig 1. Alluvial pathway from study K award toward being PI of other NIH awards

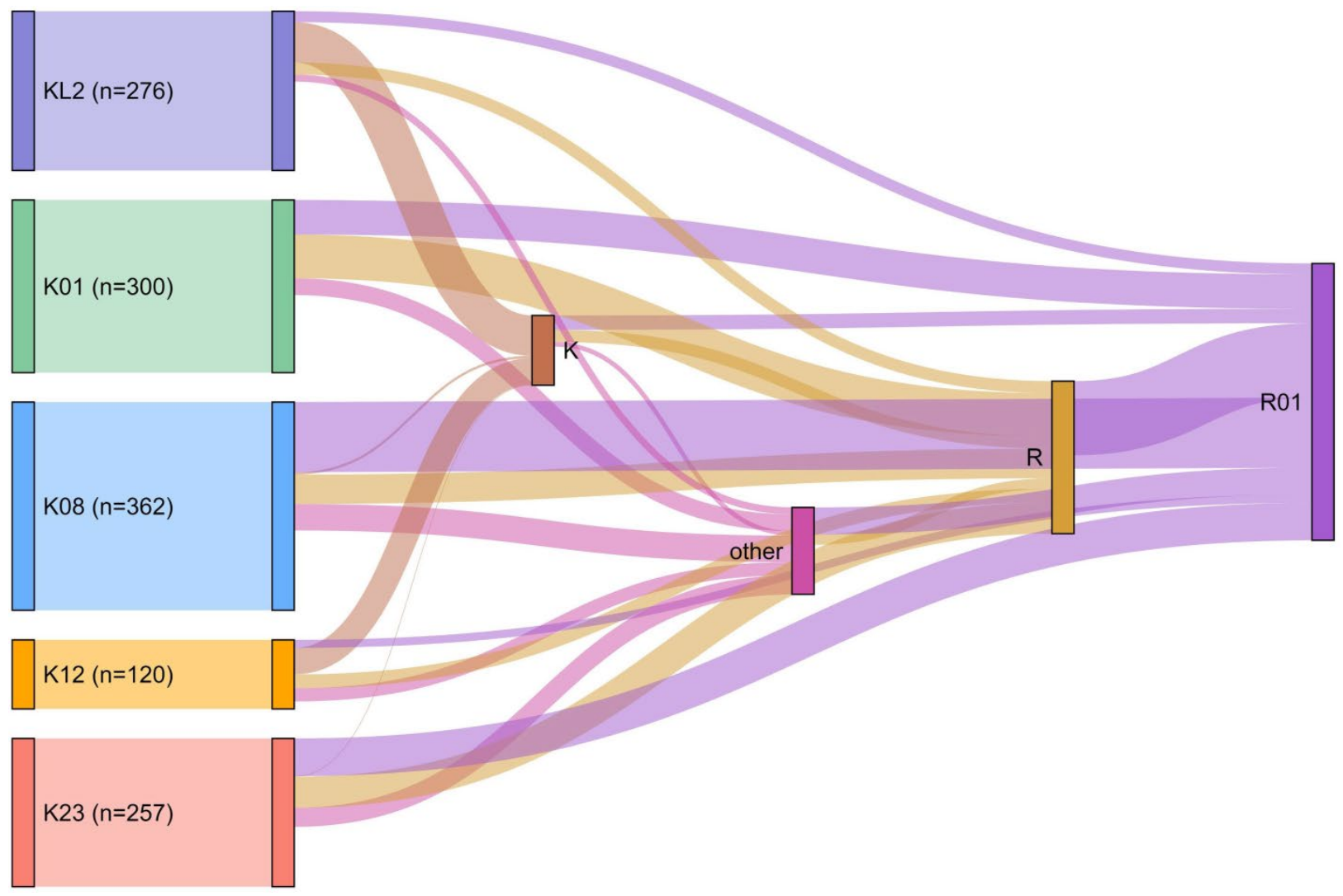

The report does not focus exclusively on outcome metrics; in Fig 1, we present a visualization of the funding pathways taken between the focal K awards and subsequent NIH awards. One detail easily obscured within a general comparison group: for the institutional $\mathrm{K}$ awardees (both the KL2 and K12), the modal next NIH award is an individual $\mathrm{K}$ award, such as 
the K08. Those already having individual K awards typically went on to either the R01 research grant or a different R, primary the R03 Small Research Grant or R21 Exploratory/Developmental Research Grant.

Finally, to step beyond indicators of research success, we used the fine-grained transaction records of UMETRICS to examine the full paid R01 teams at each university during the first year in which a study K awardee became involved — whether in a PI role or as a non-PI. Results in Table 5 suggest that the R01 teams of KL2 and comparison awardees who are coinvestigators (not PIs) are similar in size, with about 9.5 employees on average. The occupational compositions are also strikingly similar: roughly $40 \%$ faculty, $40 \%$ staff, a little over $10 \%$ students, and a little under $10 \%$ clinicians and postdoctoral researchers. When these $\mathrm{K}$ awardees go on to build their own R01 teams, however, the composition diverges. KL2 teams look much like their teams as non-PIs, minus an average of one faculty member. The comparison scholars, however, tend to lead smaller teams (an average of 6.6 employees compared to 8.3 employees per KL2 team) with a lower average staff and faculty count. These details begin to build a fuller picture of the early research career of K awardees. 
Table 5. Size and occupational composition of NIH R01 teams.

\begin{tabular}{|c|c|c|c|c|}
\hline & \multicolumn{2}{|c|}{$\mathrm{K}$ has non-PI role } & \multicolumn{2}{|c|}{$\mathrm{K}$ has PI role } \\
\hline & KL2 & Comparison & KL2 & Comparison \\
\hline \multicolumn{5}{|c|}{ Average count per team } \\
\hline Faculty & 3.7 & 3.6 & 2.8 & 2.4 \\
\hline Clinician & 0.2 & 0.2 & 0.2 & 0.1 \\
\hline Postdoctoral & 0.4 & 0.5 & 0.4 & 0.4 \\
\hline Staff & 3.9 & 3.9 & 3.9 & 2.8 \\
\hline Student & 1.2 & 1.2 & 1.0 & 0.9 \\
\hline Average team size & 9.5 & 9.4 & 8.3 & 6.6 \\
\hline $\mathrm{N}$ & 58 & 302 & 37 & 312 \\
\hline
\end{tabular}

Together, these analyses provide a window into the early careers of KL2 scholars and, crucially, a comparison group of K awardees, that CTSA hubs have been previously unable to access.

\section{Conclusion}

The aim of this pilot was to assess whether UMETRICS data and the IRIS infrastructure could be used to convert a minimal contribution from participating organizations - names of KL2 cohort members provided by CTSA hubs - into fruitful analyses of early careers in biomedical research, examining multiple dimensions with comparisons to similarly situated researchers. The three objectives identified as necessary to meet this aim produced encouraging results. First, information provided by CTSA hubs, contributed to UMETRICS by IRIS member universities, and sourced from federal databases were successfully linked together. Next, suitable comparison groups for KL2 scholars were identified among other NIH K awardees at the same 
institutions. And third, the integrated, unified data were used to examine scientific and career outcomes between awards and across universities.

Results from this pilot suggest novel means to think about the progression from a career award to an independent research career, as suggested by the variation documented in Fig 1 . Finally, the inclusion of complete data on the full—faculty, staff, student, and traineemembership of grant funded research teams enables new routes to studying faculty careers and outcomes. First, as Table 5 demonstrates, identifying instances where one faculty member is a paid collaborator on, but not a co-PI of another faculty member's grant provides a means to examine whether and how co-investigator (NIH) and senior personnel (NSF) roles on grants contribute to the development of independent faculty research careers. Second, and perhaps more importantly, information on all members of a grant-funded team allows the addition of lab or group level metrics into analyses of faculty career dynamics and outcomes. Team assembly mechanisms are associated with innovative science [47], while the structure and composition of teams is at least somewhat in the control of individual PIs. Thus, one potentially valuable direction for future research lies in deeper analyses of the ways in which team and grant staffing choices and mechanisms allow early career faculty to compose and manage teams more effectively.

Over and above the specific findings of the analyses contained in the pilot report are the implications for future efforts to examine the career and scientific effects of training interventions such as the KL2. The administrative data approach systematically assesses training outcomes with two key strengths. First, it is based on the same data across many institutions, raising comparative possibilities. Second, it is comprehensive for individuals who receive any salary support from sponsored projects. This latter feature makes it possible to examine and 
compare outcomes for many subgroups. Here that meant those receiving training in a cohortbased program and those who receive it through individual grants. For other topics, these comparisons could be made over time, between networks of collaborators, or across wholly different funding systems. Linked administrative data used in this way do have trade-offs. They cannot provide details about personal motivation, and thus offer limited insight into the ways outcomes might be shaped by, e.g., workplace climate or scholar reactions to particular training interventions. Administrative data can be taken as a complement to, not a complete replacement for, qualitative instruments or surveys such as the Rockefeller University Graduate Tracking Survey System

In summary, this pilot demonstrates that substantive evaluations of even relatively smallscale career development programs are possible using administrative data from universities with low burden on specific programs. The scale and scope of administrative data, their systematic linkage to additional information on scientific and career outcomes at the individual level, and the benefits of aggregation that can be realized with information from many different universities make rigorous evaluation of programs and program components relative to defined counterfactuals possible at a scale and cost that artisanal or survey-based methods cannot easily match. Finally, integration of research on career development outcomes for faculty offer new means to examine the effects of increasingly diverse funding trajectories, team, and collaborative network structures on outcomes as a means to advance both knowledge about the workings of science and better practices for advising and supporting early career faculty. With the IRIS infrastructure as a foundation, this approach could be extended to support rigorous multiinstitutional evaluation and research on a range of student and faculty training mechanisms. 


\section{Acknowledgements}

The authors would like to thank Sicun Chen and Najla August for their graphical and web design work on the KL2 Impact Report featured in this manuscript. They also thank Cynthia A.

Carnes, Lorah D. Dorn, Vicki Ellingrod, Shaweta Gupta, Keith A. Herzog, Rebecca D. Jackson, M. Sue O’Dorisio, Thomas E. Perorazio, Elias M. Samuels, Diane M. Thiboutot, and Emily Traw for guidance and input on this pilot study and an earlier manuscript version.

\section{References}

1. NCATS (National Center for Advancing Translational Sciences). About the CTSA Program. 2019 [cited 14 Jul 2021]. Available: https://ncats.nih.gov/ctsa/about

2. NIH Division of Biomedical Research Workforce. Research Career Development Awards | Research Training and Career Development. 2019 [cited 14 Jul 2021]. Available: https://researchtraining.nih.gov/programs/career-development

3. Schwarz N. Self-reports: How the questions shape the answers. Am Psychol. 1999;54: 93105. doi:10.1037/0003-066X.54.2.93

4. Choi BCK, Pak AWP. A catalog of biases in questionnaires. Prev Chronic Dis. 2005;2: A13. Available: http://www.ncbi.nlm.nih.gov/pubmed/15670466

5. Schwarz N, Sudman S. Autobiographical Memory and the Validity of Retrospective Reports. New York, NY: Springer New York; 1994. doi:10.1007/978-1-4612-2624-6

6. Rubio DM, Blank AE, Dozier A, Hites L, Gilliam VA, Hunt J, et al. Developing Common Metrics for the Clinical and Translational Science Awards (CTSAs): Lessons Learned. Clin Transl Sci. 2015;8: 451-459. doi:10.1111/cts.12296

7. IOM (Institute of Medicine). The CTSA Program at NIH: Opportunities for Advancing Clinical and Translational Research. The CTSA Program at NIH. Washington, DC: The National Academies Press; 2013. doi:10.17226/18323

8. CLIC (The University of Rochester Center for Leading Innovation and Collaboration). 
Common Metrics Initiative. 2019 [cited 14 Jul 2021]. Available: https://clicctsa.org/common-metrics-initiative

9. Rubio DMG. Common Metrics to Assess the Efficiency of Clinical Research. Eval Heal Prof. 2013. doi:10.1177/0163278713499586

10. NCATS (National Center for Advancing Translational Sciences). Common Metrics Initiative | National Center for Advancing Translational Sciences. 2019 [cited 14 Jul 2021]. Available: https://ncats.nih.gov/ctsa/projects/common-metrics

11. Lee LS, Pusek SN, McCormack WT, Helitzer DL, Martina CA, Dozier AM, et al. Clinical and Translational Scientist Career Success: Metrics for Evaluation. Clin Transl Sci. 2012;5: 400-407. doi:10.1111/j.1752-8062.2012.00422.x

12. Rubio DM, Primack BA, Switzer GE, Bryce CL, Seltzer DL, Kapoor WN. A comprehensive career-success model for physician-scientists. Acad Med. 2011;86: 15711576. doi:10.1097/ACM.0b013e31823592fd

13. Patel T, Rainwater J, Trochim WM, Elworth JT, Scholl L, Dave G. Opportunities for strengthening CTSA evaluation. J Clin Transl Sci. 2019;3: 59-64. doi: $10.1017 /$ cts. 2019.387

14. Tahamtan I, Safipour Afshar A, Ahamdzadeh K. Factors affecting number of citations: a comprehensive review of the literature. Scientometrics. 2016;107: 1195-1225. doi:10.1007/s11192-016-1889-2

15. MacRoberts MH, MacRoberts BR. Problems of citation analysis: A critical review. J Am Soc Inf Sci. 1989;40: 342-349. doi:10.1002/(SICI)1097-4571(198909)40:5<342::AIDASI7>3.0.CO;2-U

16. Bornmann L, Daniel HD. What do citation counts measure? A review of studies on citing behavior. J Doc. 2008;64: 45-80. doi:10.1108/00220410810844150

17. Schneider M, Kane CM, Rainwater J, Guerrero L, Tong G, Desai SR, et al. Feasibility of common bibliometrics in evaluating translational science. J Clin Transl Sci. 2017;1: 4552. doi:10.1017/cts.2016.8

18. Meyers FJ, Begg MD, Fleming M, Merchant C. Strengthening the Career Development of Clinical Translational Scientist Trainees: A Consensus Statement of the Clinical Translational Science Award (CTSA) Research Education and Career Development Committees. Clin Transl Sci. 2012;5: 132-137. doi:10.1111/j.1752-8062.2011.00392.x 
19. Shea JA, Stern DT, Klotman PE, Clayton CP, O’Hara JL, Feldman MD, et al. Career development of physician scientists: A survey of leaders in academic medicine. Am J Med. 2011;124: 779-787. doi:10.1016/j.amjmed.2011.04.004

20. Zell A, Smith L, Yanez ND, Guise J-M, Pelkey R, Ellison DH. From bedside to benchmarks: A physician-scientist workforce dashboard for biomedical research institutions. J Clin Transl Sci. 2018;2: 305-311. doi:10.1017/cts.2018.328

21. Romanick M, Ng K, Lee G, Herbert M, Coller BS. The Rockefeller University Graduate Tracking Survey System. Clin Transl Sci. 2015;8: 326-329. doi:10.1111/cts.12238

22. Schlesinger SJ, Romanick M, Tobin JN, Brassil D, Kost RG, Devine R, et al. The Rockefeller University Clinical Scholars (KL2) program 2006-2016. J Clin Transl Sci. 2017;1: 285-291. doi:10.1017/cts.2017.308

23. Sweeney C, Schwartz LS, Toto R, Merchant C, Fair AS, Gabrilove JL. Transition to Independence: Characteristics and Outcomes of Mentored Career Development (KL2) Scholars at Clinical and Translational Science Award Institutions. Acad Med. 2017;92: 556-562. doi:10.1097/ACM.0000000000001473

24. Amory JK, Louden DKN, McKinney C, Rich J, Long-Genovese S, Disis ML. Scholarly productivity and professional advancement of junior researchers receiving KL2, K23, or K08 awards at a large public research institution. J Clin Transl Sci. 2017;1: 140-143. doi: $10.1017 /$ cts. 2016.22

25. Lane J, Bertuzzi S. Research funding. Measuring the results of science investments. Science. 2011;331: 678-680. doi:10.1126/science.1201865

26. Lane J. Science innovation. Assessing the impact of science funding. Science. 2009;324: 1273-1275. doi:10.1126/science. 1175335

27. Lane J, Owen-Smith J, Rosen RF, Weinberg BA. New linked data on research investments: Scientific workforce, productivity, and public value. Res Policy. 2015;44: 1659-1671. doi:10.1016/J.RESPOL.2014.12.013

28. Teich AH. In Search of Evidence-based Science Policy: From the Endless Frontier to SciSIP. Ann Sci Technol Policy. 2018. doi:10.1561/110.00000007

29. U.S. Census Bureau. Center for Economic Studies and Research Data Centers Research Report: 2017. Washington, DC: U.S. Government Printing Office; 2018.

30. National Academies of Sciences Engineering and Medicine. The Next Generation of 
Biomedical and Behavioral Sciences Researchers: Breaking Through. Daniels R, Beninson L, editors. The Next Generation of Biomedical and Behavioral Sciences Researchers. Washington, D.C.: National Academies Press; 2018. doi:10.17226/25008

31. Fealing KH, Lane J, King JL, Johnson SR. Measuring the economic value of research: The case of food safety. Measuring the Economic Value of Research: The Case of Food Safety. Cambridge: Cambridge University Press; 2017. doi:10.1017/9781316671788

32. HHS (U.S. Department of Health and Human Services). Federal RePORTER. [cited 14 Jul 2021]. Available: https://federalreporter.nih.gov/

33. Glennon B, Lane J, Sodhi R. Money for Something: The Links between Research Funding and Innovation. SSRN. 2018 [cited 14 Jul 2021]. doi:10.2139/ssrn.3222799

34. Pezzoni M, Mairesse J, Stephan P, Lane J. Gender and the Publication Output of Graduate Students: A Case Study. PLoS One. 2016;11: e0145146. doi:10.1371/journal.pone. 0145146

35. Zolas N, Goldschlag N, Jarmin R, Stephan P, Smith JO-, Rosen RF, et al. Wrapping it up in a person: Examining employment and earnings outcomes for Ph.D. recipients. Science. 2015;350: 1367-71. doi:10.1126/science.aac5949

36. Buffington C, Cerf B, Jones C, Weinberg BA. STEM Training and Early Career Outcomes of Female and Male Graduate Students: Evidence from UMETRICS Data Linked to the 2010 Census. Am Econ Rev. 2016;106: 333-338. doi:10.1257/aer.p20161124

37. Lane J, Goldschlag N, Jarmin R, Zolas N. The Link Between University R\&D, Human Capital and Business Startups. NBER CRIW volume on The Measurement and Diffusion of Innovation. National Bureau of Economic Research; 2017.

38. Chhabra Y, Levenstein MC, Owen-Smith J. Local Fiscal Multiplier on R\&D and Science Spending: Evidence from the American Recovery and Reinvestment Act. Ross School of Business Paper No. 1383; 2019 Apr. doi:10.2139/ssrn.3201136

39. Goldschlag N, Lane J, Weinberg BA, Zolas N. Proximity and economic activity: An analysis of vendor-university transactions. J Reg Sci. 2019;59: 163-182. doi:10.1111/jors. 12397

40. Weinberg BA, Owen-Smith J, Rosen RF, Schwarz L, Allen BMF, Weiss RE, et al. Science funding and short-term economic activity. Science. 2014;344: 41-43. 
doi:10.1126/science. 1250055

41. Institute for Research on Innovation and Science (IRIS) Research Support Team. Summary Documentation for the IRIS UMETRICS 2020 Data Release. Institute for Research on Innovation and Science (IRIS); 2020. doi:10.21987/9WYN-8W21

42. NLM (U.S. National Library of Medicine). MEDLINE: Description of the Database. U.S. National Library of Medicine; 2019 [cited 14 Jul 2021]. Available:

https://www.nlm.nih.gov/bsd/medline.html

43. Kim YH, Levine AD, Nehl EJ, Walsh JP. A bibliometric measure of translational science. Scientometrics. 2020;125: 2349-2382. doi:10.1007/s11192-020-03668-2

44. USPTO (United States Patent and Trademark Office). PatentsView. In: 2018 [Internet]. 2018 [cited 14 Jul 2021]. Available: http://www.patentsview.org/

45. NCATS (National Center for Advancing Translational Sciences). Training \& Career Development. [cited 14 Jul 2021]. Available: https://ncats.nih.gov/ctsa/about/training

46. Institute for Research on Innovation and Science (IRIS) Research Support Team.

Documentation for the IRIS UMETRICS 2020 Data Release. Institute for Research on Innovation and Science (IRIS); 2020. doi:10.21987/9SAE-HR53

47. Guimerà R, Uzzi B, Spiro J, Nunes Amaral LA. Team assembly mechanisms determine collaboration network structure and team performance. Science. 2005;308: 697-702. doi: 10.1126/science. 1106340

\section{Supporting Information}

S1 Appendix. Aggregate KL2 Impact Report. This version of the report shows results from all CTSA hubs that participated in the pilot. Each hub received a report for its own university in addition to this aggregated version. 